\title{
Mediation analysis of the relationship between environmental concern and intention to adopt green concept
}

\begin{abstract}
Purpose: The purpose of this paper is to extend the theory of planned behaviour (TPB) by incorporating environmental concern (EC) and its dimensions to explain developer's intention to adopt green concepts in their future housing projects. The objectives were to investigate the relationship among the proposed constructs as well as to examine the mediating role of attitude, subjective norm and perceived behavioural control (PBC) on the relationship between EC and its dimensions and intention to adopt green concepts.

Design/methodology/approach: A survey was conducted among developer organisations in Klang Valley and the key target respondents were project managers. This study devoted priority to quantitative approach with complementary qualitative data to elaborate on the statistical results. In total, 87 usable questionnaires were returned, representing a response rate of 24.5 per cent. In addition, face-to-face interviews were carried out to gather information to complement quantitative findings.

Findings: Mediation results suggested PBC to be a significant mediator for the relationship between EC, human over nature and eco-crisis and behavioural intention. In the model between eco-crisis facet and behavioural intention, apart from PBC, attitude was found to be another significant mediator of the relationship. Interview with informants revealed that most believed human activities are causing detrimental harm to the environment. With this concern, they are confident about their organisations' ability to deliver more green projects in the future.

Originality/value: The use of specific dimension of EC and the integration of EC and its dimensions within the TPB has provided new insights to expedite the transition to sustainable construction.
\end{abstract}

Keyword: Sustainable construction; Theory of planned behaviour; Environmental concern; New ecological paradigm; Developer organizations; Pro-environmental behaviour 